\title{
CORONAL EMISSION AND STELLAR EVOLUTION
}

\author{
G. S. VAIANA \\ Osservatorio Astronomico di Palermo \\ Piazza del Parlamento 1, 90134 Palermo, Italy
}

Giuseppe S. Vaiana died untimely on August 25 1991, leaving us without a great maestro and a dear friend. We have written this abstract, based on his notes and on the many discussions we had together, as an hornage to his memory.

A. Maggio, G. Micela, S. Sciortino

\section{Evolution of Coronal Emission: Current and Foreseeable Future Knowledge}

Ideally one would like to understand the origin of coronal emission in the perspective of stellar evolution, including the role of convection, magnetic field, and angular momentum. Everyone agrees that all this has something to do with coronal emission, but to prove what mechanisms are operating in detail is a different matter.

The study of stellar X-ray coronae started a dozen years ago with the launch of the Einstein Observatory (Vaiana et al. 1981). From the beginning it emerged a richness of data, not anticipated in the original mission plans, involving the majority of stars and ages. Since then, the major developments of stellar coronal physics based on data from Einstein and EXOSAT have been reviewed (cf. for example Rosner, Golub \& Vaiana 1985; Pallavicini 1989 and reference therein). ROSAT will certainly enrich the present scenario: the improvement in coverage will eliminate some of the present biases, and the improvement in spectral resolution will contribute to a better classification of X-ray emission. Presumably, only the substantial increment in sensitivity attainable with the AXAF and XMM missions will allow other major surprises.

Presently the research on stellar coronae has two major themes: a) the study of the origin of X-ray emission, and of the variety of its manifestations; b) the implications on the integrated emission of our Galaxy and of external galaxies, on the diffuse background emission, and on the stellar counts expected from future more sensitive instruments.

Present relevant knowledge is based on the analysis of statistically representative samples selected out of optical catalogues, but with all the biases of such samples. Some of the main results can be summarized as follows:

- The X-ray luminosity decreases with stellar age, from $L_{x} \sim 10^{31} \mathrm{erg} \mathrm{s}^{-1}$ for the PMS stars down to $L_{x} \sim 10^{27} \mathrm{erg} \mathrm{s}^{-1}$ for the main sequence old disk population stars the $L_{x}$ decrease may depend on spectral type (i.e. mass; Micela et al. 1985; Barbera et al. 1991).

- The large spread of $L_{x}$ for each spectral type is largely accounted for by the mixing of stellar populations of different ages. This spread drastically reduces when considering stars of the same age, as clearly indicated by the shape of the X-ray luminosity functions (Micela et al. 1988, 1990; Barbera et al 1991). 
- $L_{x}$ by itself can be adopted as a statistical age indicator (Barbera et al. 1991; Micela, Maggio \& Vaiana 1991).

- The yellow giants as a whole show evidence of X-ray emission, while the red giants do not. The drop at $\mathrm{B}-\mathrm{V} \sim 1$ (K0) has been established on a firm statistical ground (Maggio et al. 1990). There is a suggestion of an increase of $L_{x}$ when stars leave the main sequence followed by a drop when their $B-V$ becomes $\sim 1$.

\section{Coronal Emission and Stellar Populations: the Palermo-ESA XCOUNT Galaxy Model}

XCOUNT is a numerical model for the stellar coronal components of the Galaxy (Favata et al. 1991a). From the optical point of view, it is based on the Bahcall \& Soneira Galaxy model (Bahcall 1986) which assumes the Galaxy formed by distinct disk and halo components. At the present we only model the disk component since we have little knowledge of the X-ray luminosity functions of halo stars. The few observations of halo stars in our neighborhood suggest that they are low level X-ray emitters and hence no significant contributors to $\mathrm{X}$-ray detection number. Other ingredients are the $\mathrm{X}$-ray luminosity functions of nearby stars as a function of spectral type and of stellar age (derived over the last ten years mainly by our group), and an analytic model of a galactic hydrogen density parameterizing the X-ray absorption.

As an example, we have utilized the model to predict the color distribution of the Extended Medium Sensitivity Survey stars (Favata et al., 1991b). The comparison with the observations shows: a) X-ray emission evolutionary effects which must be included to correctly predict the observed $\mathrm{dM}$ star number counts in $\mathrm{X}$-ray; $\mathrm{b}$ ) an excess of yellow stars with respect to predictions, even when known RS CVn's, W UMa, and PMS stars have been removed from the sample. The excess cannot be explained by normal giants. We suspect that the available X-ray luminosity functions of yellow stars undersample young active stars, or that a substantial fraction of EMSS yellow stars are indeed RS CVn-like.

Taken all together, these results indicate clearly that the evolutionary stage is a necessary ingredient to understand stellar X-ray emission and to properly predict, for instance, the stellar contribution to the diffuse soft X-ray background and the nature and origin of the Galactic Ridge (Micela 1991; Sciortino 1991; Micela et al. 1991).

\section{References}

Bahcall, J. 1986, ARA\&A, 24, 577

Barbera, M., Micela, G., Sciortino, S., Vaiana, G. S. 1991, Mem. SAIt, in press.

Favata, F., Micela, G., Sciortino, S., Vaiana, G.S.. 1991a, A\&A, submitted.

Favata, F., Micela, G., Sciortino, S., Vaiana, G.S.. 1991b, in "Frontiers of X-Ray Astronomy", Y. Tanaka and K. Koyama (eds), Univ. Academy Press, in press.

Maggio, A., et al. 1990, ApJ, 348, 253

Micela, G., et al. 1985, ApJ, 292, 172

Micela, G., et al. 1988, ApJ, 325, 798

Micela, G., et al. 1990, ApJ, 348, 557

Micela, G., Maggio, A., and Vaiana, G. S. 1991, ApJ, submitted.

Micela, G. 1991, Ph.D. Thesis, University of Palermo

Micela, G., Favata, F., Sciortino, S. 1991, in preparation

Pallavicini, R. 1989, A\&AR, 1, 177.

Rosner, R., Golub, L., and Vaiana, G.S. 1985, ARA\&A, 23, 413.

Sciortino, S. 1991, talk at the "Statistical Challenges in Modern Astronomy" meeting, Peen State Univ., 11-14 August

Vaiana, G. S. et al. 1981, ApJ, 245, 163 